\title{
Single Molecule Fluorescence Monitoring in Eukaryotic Cells: Intranuclear Dynamics of Splicing Factors
}

Ulrich Kubitscheck

\section{1}

\section{Motivation}

Many problems in single cell analysis involve very basic questions: where do proteins or larger molecular assemblies go? How do they move: by Brownian motion, freely or in some restricted manner, or by active transport along one of the cell's filament systems? Where are they captured, where do they bind and for how long? If binding, what are the interaction partners? Which factors are present at locations in large organelles, which are transiently bound, what is the sequence of molecular interactions? Especially the latter question is of great significance considering the functional role of large molecular complexes, which perform tasks like signal transduction, energy production, information processing, or protein formation and degradation. To approach these questions means developing an "intracellular biophysical chemistry." Currently there are only a small number of techniques available to approach these questions of intracellular protein dynamics. Light microscopy and in particular fluorescence microscopy is certainly one of the methods of choice; and it has reached a high level of maturation and sophistication. Especially the past 20 years have seen a storm of developments in quantitative fluorescence microscopic techniques, which was triggered by the perfection of microscope optics and light detectors, the widespread use of continuous wave and pulsed lasers as excitation sources, the introduction of elegant optical concepts, the availability of massive computing power to resolve complex image processing tasks and, last but not least, the introduction of genetically engineered autofluorescent protein conjugates. In the past few years tremendous progress has been made with regard to bringing optical microscope resolution almost to the ultimate level of molecular sizes with the introduction of stimulated emission depletion microscopy [1] and nonlinear structured illumination microscopy [2, 3]. But high-resolution methods are often not applicable or optimally suited to examine dynamical processes. However for such problems fluorescence techniques appear to be almost ideal. Probably the most well known is fluorescence recovery after photobleaching, abbreviated FRAP [4]. A further 
technique, nowadays almost classic but nevertheless still rapidly expanding, is fluorescence correlation spectroscopy, FCS [5, 6]. A most recent and extremely powerful technique is single molecule tracking within cells [7]. Remarkably, monitoring single fluorescent molecules with a sufficiently high time resolution can provide real-time molecular views on biochemical processes within cells even in vivo. It is extremely fascinating and instructive to directly observe the motions and interactions of single protein molecules, ribonucleoprotein particles or oligonucleotides by state of the art light microscopy.

\section{2}

\section{Experimental Approach}

Fluorescence microscopic visualization of a single molecule in real time is relatively easy to achieve if some rules are observed. Methodological prerequisites for intracellular single molecule monitoring are the reduction of background fluorescence and the use of very low concentrations of the probe of interest, which should be in the picomolar range. Technical prerequisites are the use of laser light sources to yield the required irradiance of about $0.1 \mathrm{~kW} / \mathrm{cm}^{2}$, the optimization of light transmission in the detection pathway of the microscope and finally the use of fast camera systems of utmost sensitivity for signal detection. The identification and tracking of single molecule signals in usually noisy images is finally performed using sophisticated digital image processing. Figure 1.1 shows a schematic representation of the optical

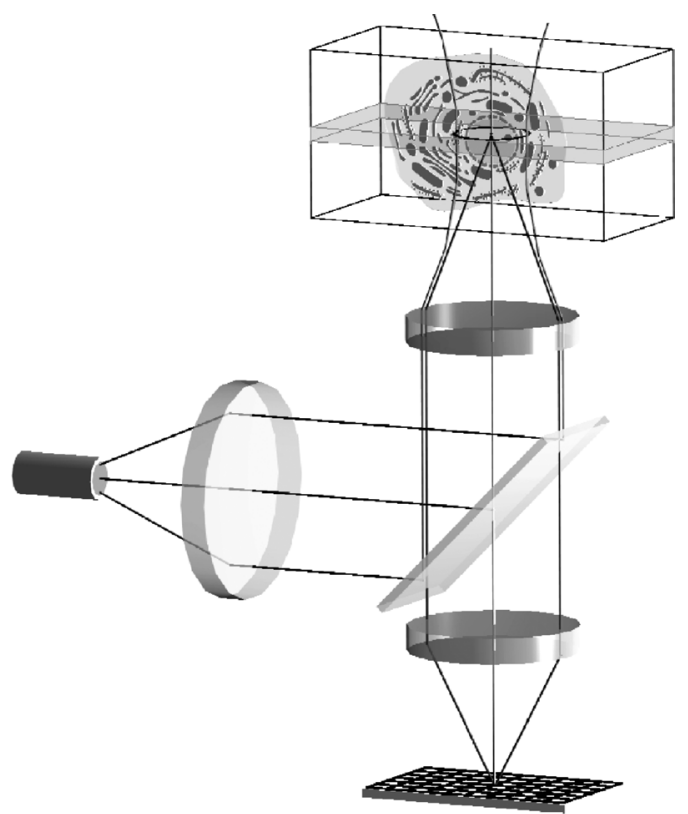

Figure 1.1 Principle optical setup of a single molecule microscope (for explanation see text). 
beam path in a single molecule microscope. The principal idea is to use as few components as possible in order to optimize the light detection efficiency.

The illumination light is coupled via an optical fiber port into the epi-illumination light path of the microscope. In this manner the collimated light illuminates the image plane, which is conjugated to the object plane and located at the position of the field stop. The beam diameter at this plane divided by the magnification of the optical system determines the final extension of the illumination field with a Gaussian intensity profile. The incoming beam is reflected towards the sample by a dichromatic beam splitter. We use a fiber output diameter of $1 \mathrm{~mm}\left(1 / \mathrm{e}^{2}\right.$ diameter $)$ and usually a $63 \times$ objective lens. This produces a Gaussian illumination pattern with a diameter of approximately $16 \mu \mathrm{m}$ within the object plane. Currently, we study preferentially dynamic processes within cell nuclei; and therefore the illumination field size is adjusted approximately to the diameter of a single nucleus. The focal depth, that is the axial extension of the object area in focus, is less than $1 \mu \mathrm{m}$ for high end objective lenses with a numerical aperture (NA) $\geq 1$. Since the light collection efficiency of the objective is proportional to the square of the NA, the lens with the highest NA available should be chosen. When the laser is focused onto the back focal plane of the objective lens, a homogeneous illumination of the complete object field can be achieved. The fluorescence image is captured by the objective lens, transmitted by the dichromatic beam splitter and finally mapped by the tube lens onto the charged coupled device (CCD) of the camera. In order to achieve the highest frame rates and sensitivity available, a back-illuminated electron multiplying (EM) camera chip with $128 \times 128$ pixels is a suitable choice as imaging device. After careful optimization of all components in the detection path of the microscope, an overall detection efficiency in the range of $10 \%$ is achievable. Figure 1.2 shows more technical detail of the beam path within the microscope. We use Notch filters in the emission beam path instead of the commonly employed band pass filters.

These reject any residual excitation light with an optical density (OD) of 6 OD units which might pass the dichromatic mirror. Therefore, no Raleigh scattered light reaches the detector. The Notch filters are positioned within the infinity beam path in front of the tube lens. A set of three different Notch filters centered at 488, 532 and $633 \mathrm{~nm}$ is available and, depending on the employed laser excitation, the respective filter is inserted. The tube lens focuses the image but it is supplemented by a $4 \times$ magnifying lens system. This is required because our camera (iXon DV-860-BI; Andor Technologies, Belfast, Northern Ireland) has a pixel size of $24 \mu \mathrm{m}$. Used without a magnifier this would correspond to a pixel size in the object plane of $380 \mathrm{~nm}$, which would not be sufficient for satisfactory detail when imaging diffraction-limited objects. Microscopic imaging of single fluorescent molecules produces diffraction-limited light spots in the image plane. The corresponding intensity pattern can be approximated by a two-dimensional Gaussian function with a full width at half maximum of approximately $250 \mathrm{~nm}$, when light with a wavelength of $500 \mathrm{~nm}$ and an objective lens of NA 1.4 are used for imaging. The precise position of the molecule can therefore be obtained by a fitting process which yields the center of the light spot with a very high precision, but only if the single molecule signal is imaged with a good signal to noise ratio and sufficient geometric detail. The required 


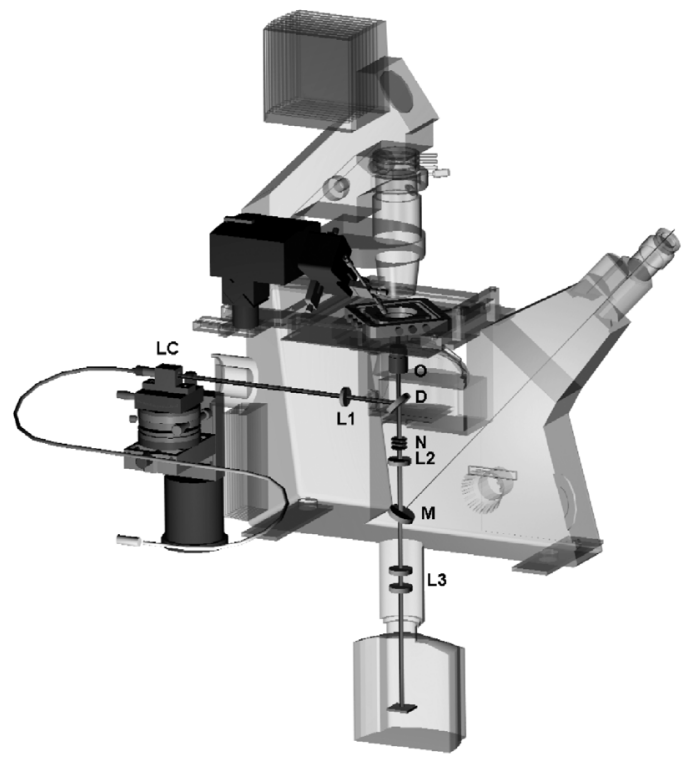

Figure 1.2 Details of the single molecule microscope. LC, fiber output; L1, tube lens in the illumination light path; L1, tube lens in the emission light path; L3, $4 \times$ magnifying lens system; O, objective lens; D, dichromatic beam splitter; N, Notch filters.

detail is obtained when a $4 \times$ magnifier is used, which reduces the object plane pixel size to well below the diffraction limit, namely to $95 \mathrm{~nm}$. Then a diffraction-limited single molecule signal is spread over about $5 \times 5$ pixels, which is ideal for nanolocalization by the fitting process. The localization precision depends only on the signal to noise ratio (SNR) and the stability of the optical setup; values of $2-40 \mathrm{~nm}$ can be achieved [8-10].

Figure 1.3 gives a full schematic view of the complete setup, showing the details of the excitation laser setup. Our instrument allows the sequential use of up to three different laser lines for fluorescence excitation. The different laser beams are combined by two dichromatic beam splitters, then coupled into an acousto-optical tunable filter and focused into an optical mono-mode fiber. This delivers the light to the microscope. As outlined above, the triple dichromatic beam splitter reflects the three excitation lines onto the sample, while the three fluorescence bands are transmitted to the detection system via the Notch filters. This slightly unusual filter construction has the following purpose: by this means three fluorescence bands can be detected independently by a single EM CCD camera by simply alternatively triggering the AOTF driver governing the laser illumination. The camera generates the trigger signals whenever an image is acquired. Using a single camera for the imaging of the different fluorescence channels instead of using several cameras separated by additional emission beam path dichromatic beam splitters has the great advantage that alignment of the fluorescence images is not required, since registration is realized 


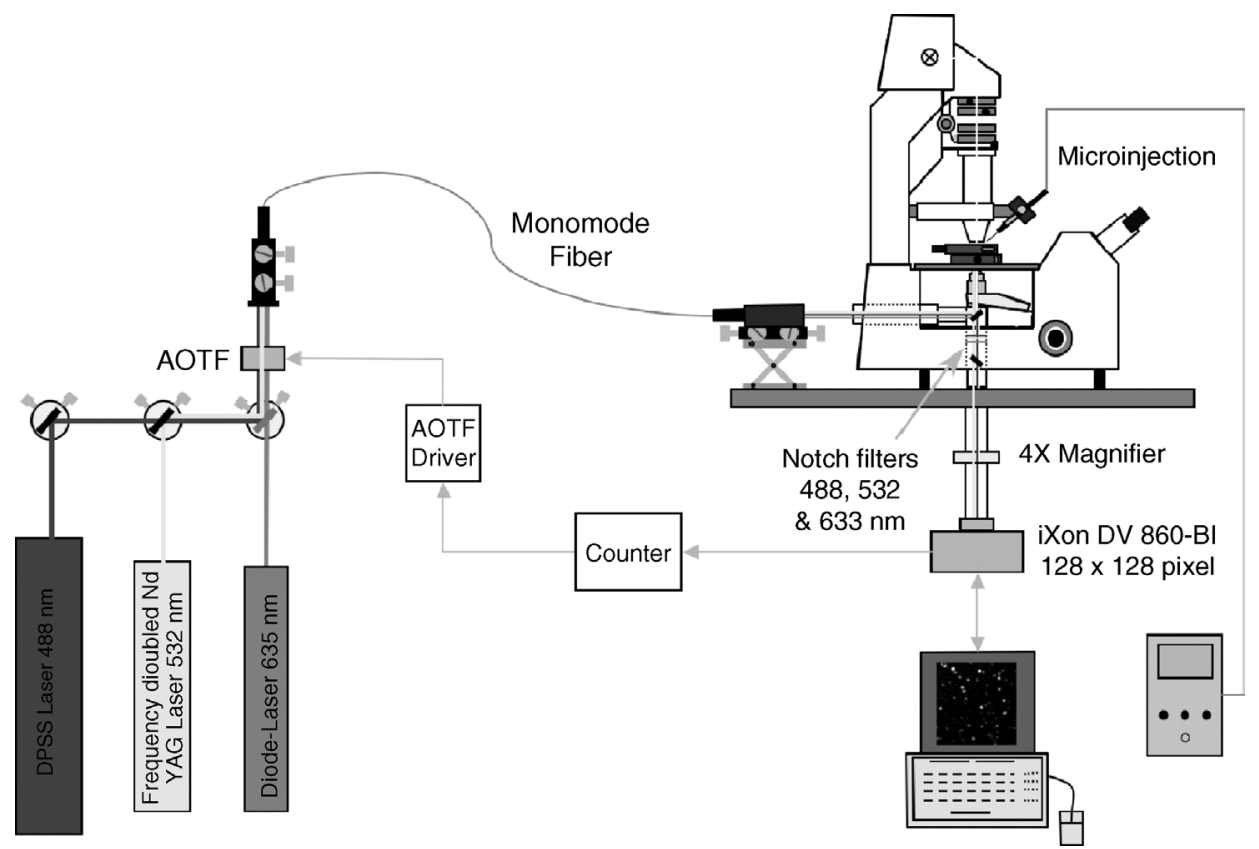

Figure 1.3 Sketch of the single molecule microscope including the excitation lasers. Three excitation laser lines were joined by two dichromatic beam splitters and passed through an acousto-optical tunable filter (AOTF), which allowed regulating the power for each laser separately within a few microseconds. Excitation light was directed to the inverted microscope by an optical mono-mode fiber, and coupled in at the epi-illumination port. The emitted fluorescence light was separated from the excitation by a triple dichromatic beam splitter, which reflected the three excitation lines onto the probe, while the three fluorescence bands were transmitted to the detection light pass by optionally insertable Notch filters with an absorbance of OD 6 at the laser lines. No further filters were used in the emission beam path. Hence, up to three fluorescence bands could be detected sequentially by a single electronmultiplying CCD camera by alternative triggering the AOTF driver via a programmable counter, which reacted to the camera-generated trigger signals. Fluorescent probe molecules could be microinjected into living cells.

in the very same beam path. Just minor lateral and axial chromatic aberration of the detection optics need be taken into account.

The most straightforward way to deliver a fluorescent probe molecule into living cells is by using a microinjection system. Intracellular fluorescent probe concentrations should be adjusted to $<100 \mathrm{pM}$, since then only a few fluorescent molecule are present within the field of view. For extended observations, the molecules must be and move within the focal region of the objective lens. Signals from molecules leaving the focal plane rapidly vanish into the background noise, which means that the imaging of single point objects exhibits a three-dimensional resolution.

The observation of single molecules with high imaging frame rates allows the tracing of single molecule trajectories, if their movement during image acquisition is negligible. It has been shown that frame rates in the range of $300-400 \mathrm{~Hz}$ are 
sufficient to follow the pathways of protein molecules with molecular masses above $50 \mathrm{kDa}$ in solution [11]. In that study it was shown for two model proteins streptavidin and an IgG - that the quantification of diffusion based on tracing single protein molecules in aqueous buffer yielded the same results as the respective measurements using fluorescence correlation spectroscopy (FCS). Also, it was demonstrated that the high-speed video microscope employed could easily follow single fluorescent quantum dots. Hence, for molecules in the respective molecular mass and brightness range, single molecule tracking may be used as a complementary method to FCS for analyzing protein mobility. Tracer molecule mobility within biological cells is four- to 10 -fold reduced, compared to mobility in aqueous solution [4]. Thus, Grünwald et al. showed that real-time visualization and tracking of protein molecules inside living cells is feasible using a state of the art imaging system.

\section{3}

\section{Single Particle Tracking within Living Cells}

Several recent studies demonstrated the power of single molecule tracing within living cells [12-16]. Of course, the technique is not restricted to imaging single molecules. Actually, it is even more straightforward to image other subdiffraction sized objects, like quantum dots, single viruses or single gene carriers, because they are usually moving more slowly and can often be labeled with a higher fluorophore to particle ratio [17-21]. The first successful observations of single proteins inside cells were performed using large autofluorescent proteins as probes for intracellular mobility [22]. The effective intracellular viscosity experienced by these probes was found to be about 15 -fold higher than that of an aqueous solution, but with a much broader distribution of diffusion constants. The large range of protein mobility is obviously due to interactions with intracellular components or macromolecules and organelles forming barriers and obstacles.

When considering single molecules the tracking of molecules and particles is especially feasible within the cell nucleus, since the autofluorescence of the cell nucleus is significantly lower than that of the cytoplasm [13]. Single molecule observation within cell nuclei allows the analysis of nucleocytoplasmic transport and the characterization of intranuclear transport pathways, mobility restrictions and intranuclear binding processes. The past 10 years revealed that the nuclear architecture is much more complex than previously thought. The new view is that the cell nucleus is a highly organized organelle in which chromosomes occupy distinct territories and processes like DNA transcription and replication as well as mRNA splicing are carried out by supramolecular complexes which are organized in complex spatio-temporal patterns [23]. Even inert protein probes, which do not specifically interact with nuclear structures, do not perform free Brownian motion but rather exhibit various mobility modi (Grünwald, Martin, Buschmann, Leonhardt, Kubitscheck and Cardoso, unpublished data). Diffusion of such molecules is generally characterized by anomalous behavior, that is the effective mean square 
displacements do not depend linearly on time as for normal Brownian motion. The analysis of single molecule trajectories confirms the existence of very large restrictions on the mobility of large proteins within the cell nucleus, which is indicative of structural barriers and frequent binding-unbinding events to immobile or slowly moving supramolecular structures.

\section{4}

\section{Pre-Messenger RNA Splicing}

Molecules interacting specifically with further intranuclear components such as DNA, RNA or protein complexes exhibit a very different mobility in comparison to inert ones. In this case a detailed mobility analysis allows insight into the dynamics of the corresponding intranuclear processes.

Eukaryotic RNA transcripts go through several post-transcriptional modifications before their final transport into the cytoplasm [24]. Usually such pre-messenger RNAs have noncoding sequences - so-called introns - that must be removed to yield functional mRNA. This biochemical processing is designated as "splicing." It is a complex intranuclear process accomplished by pre-assembled complexes, the "spliceosomes," which comprise more than 70 different proteins. Many of these are contained in the uridine-rich small nuclear ribonucleoprotein particles (U snRNPs), which are classified as U1, U2, U5 and U4/U6 according to their small nuclear RNA (snRNA) content. With the exception of U6, the snRNAs are synthesized in the nucleus by RNA polymerase II and exported to the cytoplasm, where sets of common and specific proteins bind to the snRNAs [25]. After their assembly the U snRNPs are imported into the nucleus. Spliceosomes have been shown to be subcomplexes of huge multicomponent nuclear RNP complexes, so-called supraspliceosomes with geometric extensions of $50 \times 50 \times 35 \mathrm{~nm}^{3}$ [26]. The group of proteins and RNPs involved in splicing is collectively designated as splicing factors. The intranuclear distribution of splicing factors is a prominent example of the high degree of spatio-temporal organization of the nuclear contents. Immuno-staining of cells with antibodies against splicing factors such as ASF/SF2 or U snRNPs produces images in which nuclei contain numerous tiny bright spots, the so-called "nuclear speckles", dispersed on a homogeneous background. These speckles are created by the enrichment of splicing factors in interchromatin granule clusters and perichromatin fibrils, which are designated as splicing factor compartments [27]. The mechanism of their formation and their function is still unresolved. They may represent sites at which splicing factors are reprocessed, stored or assembled together with other components of the transcription and RNA processing machinery into spliceosomes. It has been speculated that they arise by interaction with a putative karyoskeleton, or alternatively by self-assembly. The diffuse nucleoplasmic staining is probably due to unbound splicing factors and those actively involved in splicing. Of course, it is most interesting to study the dynamical behavior of splicing factors within and outside speckles in order to get insights into the different processes taking place in these intranuclear domains. 
1.5

Intranuclear Splicing Factor Tracking

In a recent study we analyzed the mobility of native U1 snRNPs within live cells at the single particle level in order to yield insight into the dynamics of mRNA splicing [16]. U1 snRNPs were isolated from HeLa cell nucleus lysate by ultracentrifugation and then covalently labeled with the red fluorescent dye Cy5. The use of native RNPs excluded possible problems of particles that were assembled in vitro and might be incomplete or functionally imperfect. The Cy5-labeled U1 snRNPs were microinjected into the cytoplasm of living HeLa cells and were subsequently imported into the cell nuclei. Thereby the biochemical functions, integrity and structure of the nuclei remained as unaffected as possible. The concentration of the injection solution chosen was so low that single U1 snRNPs could be visualized and tracked within the cell nuclei with a spatial precision of roughly $30 \mathrm{~nm}$ by the single molecule microscope described above. In this manner movies from single U1 snRNPs with different time resolution ranging from $200 \mathrm{~Hz}$ to $5 \mathrm{~Hz}$ were recorded. The HeLa cells employed were transiently transfected with ASF/SF2-GFP in order to allow the study of the RNP dynamics within and outside nuclear speckles. Images of these reference structures were taken using GFP fluorescence excitation at $488 \mathrm{~nm}$, while the single RNP trajectories were obtained switching laser excitation to $632 \mathrm{~nm}$ for excitation of Cy5. Automatic image analysis procedures on the basis of Diatrack 3.01 (SemaSopht, North Epping, Australia) were employed to identify single RNP signals and to reconstruct single particle trajectories (Figure 1.4).

These could be analyzed depending on their assignment to speckled or nucleoplasmic intranuclear domains. The greater fraction of the detected U1 snRNPs was immobile: roughly $80 \%$ of the U1 snRNPs identified in two subsequent frames did not move beyond the localization precision and therefore were classified as immobile. Such immobilization may be caused by binding to a very large and bulky structure with no or extremely low mobility. Evidently, being coupled to large intranuclear complexes is the standard state of U1 snRNPs.

This observation was in strong contrast to the results of a related single molecule tracking study, in which an inert probe molecule - streptavidin-cy5 - was tracked inside cell nuclei (D. Grünwald, R. Martin, V. Buschmann, H. Leonhardt, U. Kubitscheck and M.C. Cardoso, unpublished data). In that case, a significantly smaller fraction of molecules, namely about $20 \%$, was immobile between successive frames. Furthermore, the duration of binding was significantly shorter in these experiments.

Presumably the observed immobilization of the U1 snRNPs was often caused by the involvement of the particles in ongoing splicing events. This assumption was suggested by comparing the results of a previous study on intranuclear U1 snRNP mobility in our laboratory, which was performed not on living but rather on digitoninpermeabilized cells [28]. Digitonin-permeabilized cells are mostly physiologically inactive and splicing has ceased or is to a very large extent reduced. Notably, in those cells a significantly smaller fraction of RNPs was found immobile, namely only about $20 \%$, as in the case of the unfunctional tracer molecule streptavidin. It is well known 

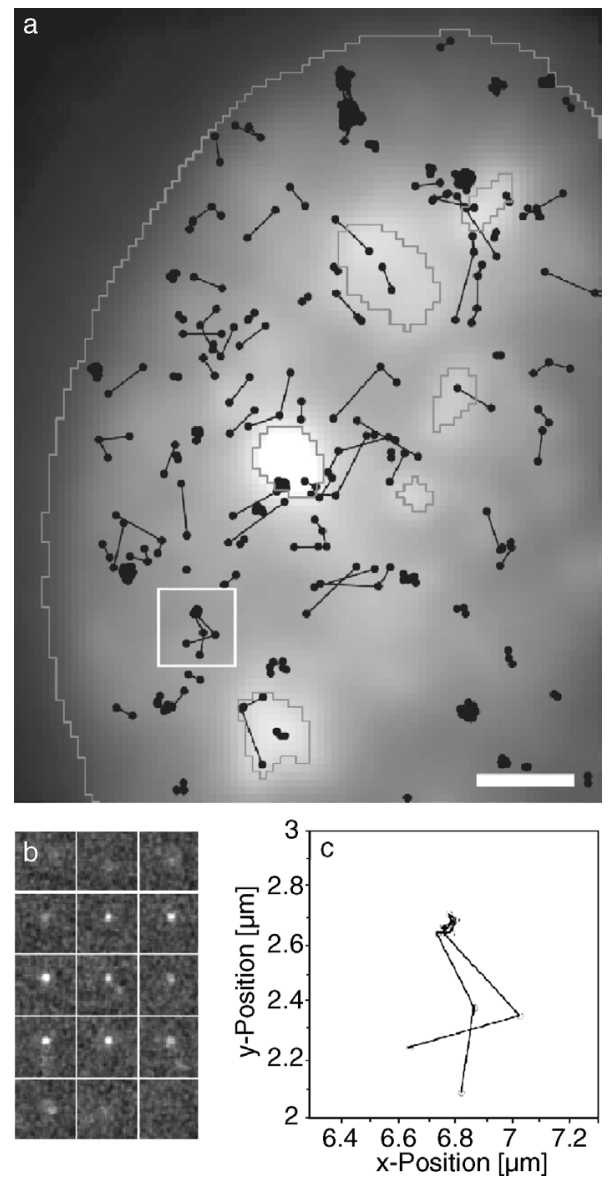

Figure 1.4 Trajectories of single U 1 snRNPs in a The white line indicates the estimated position of living cell nucleus. (a) Trajectories of numerous the nuclear envelope (bar: $2 \mu \mathrm{m}$ ). (b) The short single U1snRNPs extracted from a single image sequence of the trajectory marked in (a). molecule movie, which was recorded at $200 \mathrm{~Hz}$. The series demonstrates the excellent signal to In the movie, mobile U1 snRNPs as well as transiently immobilized RNPs could well be noise ratio of the labeled U1 snRNPs. The sequence starts with the image in the upper left distinguished within nucleoplasm and speckles. hand corner, and goes linewise to the lower right The black dots connected by lines represent the positions and molecular jumps as identified by Diatrack 3.0. The trajectories were plotted hand corner. Single frame integration time was $10 \mathrm{~ms}$; single image size $1 \mu \mathrm{m}^{2}$. (c) Magnified view of the positions at which the U 1 snRNP was observed, revealing that the RNP moved to a onto a gray-scale image of the splicing factor ASF/SF2-GFP, which was transiently expressed in the respective HeLa cell nucleus. Hence, the bright regions indicate nuclear speckles (dark gray lines). The white box marks a specific site, was bound there for $80 \mathrm{~ms}$, and then left the site again; complete field size, $1 \mu \mathrm{m}^{2}$. The figure is modified from Ref. [16] and is shown with permission. single trajectory which is detailed in (b) and (c). 
that pre-mRNA splicing, at least in part, is already occurring during transcription. This means that very large DNA-pre-mRNA-protein complexes are formed the transcription sites, which would certainly represent large supramolecular complexes causing a gross immobilization of involved interaction partners - such as the U1 snRNPs.

\section{6}

\section{Intranuclear U1 snRNP Splicing Factor Binding}

The recorded movies also allowed a study of dissociation from the putative binding sites - simply by counting the number of frames after which the respective particles vanished from their fixed positions. Of course, the disappearance could in principle also be due to photobleaching of the Cy5-labeled RNPs, but it was found that the average photostability of the particles was not a limiting factor.

In the case of a simple bimolecular dissociation reaction one would expect a monoexponential decay of the number of complexes. In contrast to that, dissociation from the binding sites showed unexpected kinetics. Using movies taken with high frame rates to analyze the binding revealed short binding durations, while movies with low frame rate indicated long binding durations. In this manner dissociation times between $5 \mathrm{~ms}$ and $1400 \mathrm{~ms}$ were determined, ranging over three orders of magnitude. We interpret this result in the following manner. The complex dissociation kinetics most probably reflect the different ways in which U1 snRNPs interacted with other nuclear structures. We assume that we observed processes ranging from nonspecific interactions or "trapping" within a chromatin network - as in the case of nonfunctional streptavidin - and genuine splicing events to the assembly of spliceosomes before splicing and possibly the re-assembly of splicing factors. Furthermore, numerous quite diverse types of splicing reactions occur simultaneously within a cell nucleus, for example involving a few or numerous introns either in a single pre-mRNA reaction or in alternative splicing reactions. It is plausible that all of these display a different kinetics with regard to the duration of the involvement of different splicing factors. Therefore the observation of many different types of kinetics is probably not incidental but displays a fundamental property of intranuclear reaction kinetics. It is tempting to speculate that chemical kinetics occurring on many time scales is typical for the complex processes occurring in cell nuclei.

\section{7}

\section{Events in Speckles}

Speckles can in vivo be defined using cells expressing ASF/SF2-GFP conjugates. Then, distinct spots of strong GFP fluorescence mark the position of speckles, which appear quite contrasted in a confocal microscope. Acquisition of images upon green excitation in nonconfocal video images also allowed discrimination between speckles and nucleoplasmic spaces and created a basis for a separate analysis of U1 snRNP 
dynamics in speckles and in the nucleoplasm. Surprisingly, the dissociation kinetics did not differ significantly for binding within or outside speckles. This indicated that the increased splicing factor concentration in speckles is not due to a stronger binding of U1 snRNPs. Rather, two explanations for the higher concentrations of splicing factors within speckles remain: (i) the on rate of the binding process is increased, for example by a facilitated accessibility of the speckle binding sites, (ii) the concentration of binding sites within the speckles is higher than in the remaining nucleoplasmic space, while the interaction is of similar nature. The data presently available do not allow discrimination between the two options. Above, we presented arguments that at least part of the observed binding was related to splicing during ongoing transcription. Therefore it can be assumed that mRNA transcription and splicing also takes place in speckles, because the processes causing U1 snRNP immobilization are identical inside and outside the speckles. In summary, the reason for the complex immobilization pattern cannot yet be explained. Nevertheless, it is clear that the study of complex physiological processes such as transcription and splicing require further live cell measurements.

\section{8}

\section{Intranuclear U1 snRNP Mobility}

Obviously, single particle tracking is ideal for recording molecular trajectories (Saxton and Jacobson, 1997). Above, we discussed that a frame rate of $350 \mathrm{~Hz}$ is sufficient to track single protein molecules in buffer solution. Therefore, a repetition rate of $200 \mathrm{~Hz}$ should be high enough to record real-time U1 snRNP trajectories in the cellular interior. There exist several approaches to analyze single molecule tracks.

The trajectory for each U1 snRNP was determined by Diatrack as a set of coordinates $\left\{x_{i}, y_{i}\right\}$, where $1 \leq i \leq N$ with $N$ denoting the number of observations. From each trajectory a total of $(N-1) \cdot N / 2$ square displacements, $r^{2}\left(t_{\mathrm{lag}}\right)$, was determined between two positions separated by a time lag, $t_{\mathrm{lag}}$, with $t_{\mathrm{lag}}=n\left(t_{\mathrm{ill}}+\right.$ $\left.t_{\text {delay }}\right) \cdot t_{\text {ill }}$ denotes the single frame integration time, $t_{\text {delay }}$ the delay time between two successive frames and $n$ the difference between the frame numbers. By averaging the square displacements for equal to $t_{\text {lag }}$ the mean square displacements (MSD), $\left\langle r^{2}\left(t_{\text {lag }}\right)\right\rangle$ can be calculated. In the case of two-dimensional Brownian motion the diffusion coefficient $D$ is related to the MSD by:

$$
\left\langle r^{2}\left(t_{\mathrm{lag}}\right)\right\rangle=4 D t_{\mathrm{lag}}
$$

Thus, a linear relationship between MSD and lag time indicates unrestricted Brownian motion and can be used to derive diffusion coefficients from single or many trajectories. But if the observed molecular motion is not based on free diffusion but on confined diffusion or directed flow, the relation between $\operatorname{MSD}\left(t_{\mathrm{lag}}\right)$ and $t_{\mathrm{lag}}$ is no longer linear. Analysis of trajectories by Equation 1.1 is also not appropriate, when the particles have a different mobility, possibly due to interactions with further molecules, or when particles change their mode of motion along a trajectory. In such cases the data can be analyzed by so-called jump distance distributions. This analysis 
considers the probability $p(r, t) d r$ that a particle starting at some arbitrary position is encountered within a distance between $r$ and $r+d r$ from the starting point at a given, later time $t$. For particles diffusing in two dimensions the jump distance distribution can be written as:

$$
p(r, t) d r=\frac{1}{4 \pi D t} e^{-r^{2} / 4 D t} 2 \pi r d r
$$

Experimentally this probability distribution can be approximated by a normalized frequency distribution obtained by counting the jump distances within respective intervals $[r, r+d r]$ covered by single particles after a given number of frames respective to the lag times. The essential point in the jump distance distribution analysis is that subpopulations of jumps can be determined by curve fitting.

So far, the mobility of single molecules within cell nuclei cannot be characterized by simple Brownian motion. This was demonstrated by quite a number of previous studies using FCS and single particle tracking [13, 22, 29]. In our study on U1 snRNP dynamics, we had to discriminate one immobile and at least two mobile fractions of molecular jumps. Hence, a satisfactory fit of the data required three terms like that given in Equation 1.2 (Figure 1.5).

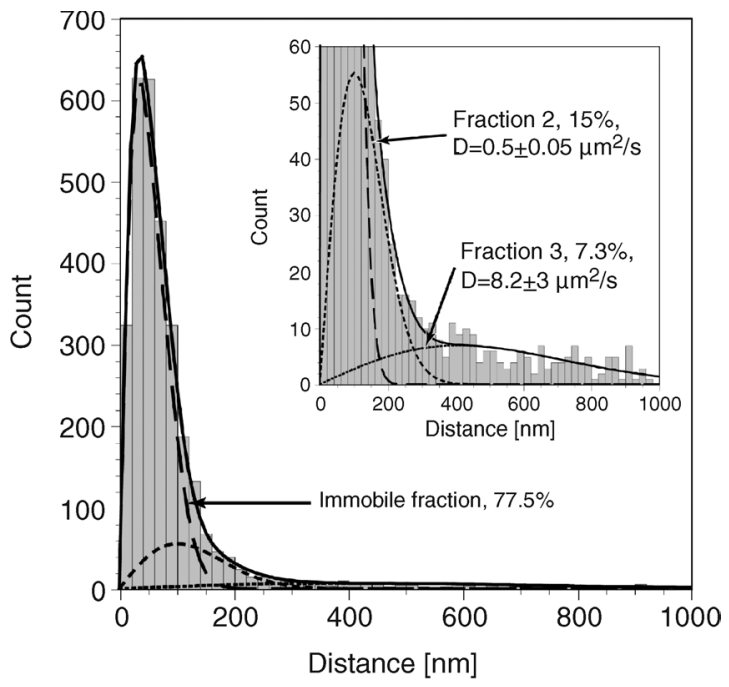

Figure 1.5 Motion analysis of single U1 snRNPs in successive frames. Distribution of the precision. Two mobile jump fractions, $f_{2}$ and $f_{3}$, corresponded to fast diffusional motion with distances covered by single, nucleoplasmic U1 snRNPs, which were identified and tracked in successive frames of movies acquired at a frame rate of $100 \mathrm{~Hz}$. A minimum number of three different diffusion terms was needed for a coefficients of $D_{2}=0.51 \pm 0.05 \mu \mathrm{m}^{2} / \mathrm{s}$ (short dashes, $15 \%$ ) and $D_{3}=8.2 \pm 3 \mu \mathrm{m}^{2} / \mathrm{s}$ (dotted line, $7.3 \%$ ). The sum of all three fractions excellently described the data (full line). The insert shows the same plot with a different satisfactory fit of the data. The greatest fraction of scaling of the ordinate to show the great number jumps (long dashes, 77.5\%) corresponded to of large jumps corresponding to $D_{3}$. The figure immobile particles. These performed only a was taken from Ref. [16] and is shown with seeming movement due to the finite localization permission. 
The dissection of the distribution into three fractions represented a minimum number, meaning that three fractions were sufficient to fit the jump distance distribution. Certainly the fit could have been optimized by using four or five fractions, or even a continuous distribution. Furthermore it is important to note that the different jump fractions do not correspond to different particles with distinct mobilities. Rather - as could be noted in many trajectories - single particles switch their mode of motion along their trajectory, for example from mobile to bound or back. This is no surprising observation considering the complex intranuclear dynamics. Hence the fractions identified in the jump distance histograms also represent different modes of motion of possibly identical particles. Actually, we suppose that distinct mobility fractions do not exist, but rather that the U1 snRNP mobility can be characterized by motions which range from immobility up to dynamics which can be characterized by a diffusion coefficient of $8 \mu \mathrm{m}^{2} / \mathrm{s}$.

The mobile U1 snRNPs showed diffusion coefficients varying from $0.5 \mu \mathrm{m}^{2} / \mathrm{s}$ to $8.0 \mu \mathrm{m}^{2} / \mathrm{s}$. A diffusion coefficient of $8 \mu \mathrm{m}^{2} / \mathrm{s}$ is four- to fivefold lower than that expected for a $240-\mathrm{kDa}$ particle such as U1 snRNP in aqueous solution. A fourfold reduction in mobility was found in several previous mobility studies of tracer molecules within cell nuclei, as already stated. Therefore we suppose that this diffusion coefficient corresponds to the motion of uncomplexed U1 snRNPs moving within nuclei, as in a solution with an effective viscosity of $5 \mathrm{cPoise}$. It has been shown that $U$ snRNPs are central components of pre-formed complexes designated as spliceosomes and even supraspliceosomes, which contain four native spliceosomes with a total mass of $21 \mathrm{MDa}$ and geometric dimensions of $50 \times 50 \times 35 \mathrm{~nm}^{3}$ [26]. For such complexes, the Stokes-Einstein law predicts a diffusion constant of $D \approx 2 \mu \mathrm{m}^{2} / \mathrm{s}$ in a solution of 5 cPoise. Obviously this value perfectly lies within the range of diffusion constants determined for U1 snRNPs. However huge complexes such as supraspliceosomes would probably not move in an unrestricted manner within the molecular crowded and entangled intranuclear space. They would suffer multiple collisions per unit time with chromatin or other molecular complexes, which would slow it down even further. Exactly this has been observed for large $2 \mathrm{MDa}$ dextran molecules. Their mobility was dependent on the concentration of intracellular obstacles [30]. Furthermore it has been shown that chromatin regions represent a significant obstruction for the accessibility of large probe molecules [31]. Hence jumps corresponding to a mobility as low as $D=0.5 \mu \mathrm{m}^{2} / \mathrm{s}$ could well correspond to U1 snRNPs contained in supraspliceosomes moving in a strongly hindered manner through a crowded nucleoplasm.

\section{9}

\section{Perspectives of Single Molecule Microscopy}

Single molecule visualization and tracking is especially advantageous in single cell analysis for a number of reasons. Many signaling processes in biological systems are triggered by a very small number of effector molecules which can easily escape the classic methods of detection. Single molecule microscopy requires exceedingly low 
probe concentrations and hence is ideally suited to study such events. In addition the use of low probe concentrations guarantees that the physiological processes in investigation are only minimally disturbed. Processes like RNA splicing or transport through the nuclear pore complex take place on length scales between molecular distances $(0.1-1.0 \mathrm{~nm})$ and the optical microscope resolution limit $(>250 \mathrm{~nm})$. Actions on such "intermediate-sized" length scales cannot directly be studied with conventional microscopy. Furthermore they usually involve large supramolecular complexes containing numerous protein molecules and RNPs, which makes their reconstitution in vitro very difficult. Single molecule detection within live cells with a spatial precision in the nanometer range combined with the simultaneous use of multiple fluorescent labels and exploitation of single molecule energy transfer is a suitable way to approach such complex problems.

The molecular dynamics during ongoing live processes within cell nuclei have often been measured by photobleaching or photoactivation techniques or FCS. In order to correctly account for the mobility data obtained, binding processes are postulated [32]. Alternatively anomalous diffusion models can also adequately describe the data [29]. In contrast, binding events do not have to be assumed in single molecule tracking, but rather can be directly observed and discriminated from molecular movements. It is possible not only to observe distinct binding events but also to measure the duration of individual interactions. Dissociation data like that discussed above for U1 snRNPs can usually be obtained only by a direct preparation of the molecular complexes in the initial, associated state. This often presents a problem which is very difficult or impossible to solve, especially when working in vivo. Obviously single molecule detection elegantly solves this problem, because the individual events can be aligned in time a posteriori. The observation of individual molecular interactions does not require synchronization of the molecular ensemble. Finally in contrast to conventional techniques, such as FCS or photobleaching techniques, different forms of mobility in heterogeneous systems may well be discriminated by single molecule tracking. It is especially well suited for dynamic processes with diffusion coefficients $<10 \mu \mathrm{m}^{2} / \mathrm{s}$.

Two methodological drawbacks can currently be identified. As a video microscopy the technique lacks a true optical sectioning capacity. Although the imaging of single particles provides an intrinsic three-dimensional resolution, molecules in the out of focus regions are excited and drastically reduce the signal to noise ratio of the molecules in focus and thus lower the respective localization precision. Furthermore the reference structures, which must be imaged in order to locate the dynamic single molecule processes within a cellular context, appear blurred in current applications. However an elegant solution to this problem can be imagined. The sample may be illuminated using a light sheet perpendicular to the observation axis. This yields an intrinsic optical sectioning effect [33-35]. This clever illumination principle can be incorporated without major problems in a high-speed video microscope with single molecule sensitivity. Finally a bottleneck is still represented by the image processing of single molecule movies. Inherently noisy single molecule signals must be identified in thousands of images and the true trajectories must be constructed out of the single, fitted positions. Obviously this is often not possible in an unambiguous 
manner and sophisticated numerical approaches must be applied to extract a maximum of information from the data. A very small number of commercial program packages are currently available for this task, but none of them is able to cope with the data in a really satisfactory manner. The development of suitable modern image analysis techniques to this problem would really produce a significant progress for the field.

The conventional way to analyze intracellular mobility is by FRAP, which measures bulk mobility on a spatial scale of several microns within a time window of up to $50 \mathrm{~s}$. Currently, FCS is increasingly used to study intracellular dynamics at selected focal positions. Single molecule tracking quantifies the mobility of individual molecules on length scales from $20 \mathrm{~nm}$ up to several microns within time windows from milliseconds to seconds. It is not at all straightforward to extrapolate from single molecule data to the results of FCS or FRAP measurements and vice versa. We think that it would be most fruitful to carefully apply all three techniques in a complementary manner and to correlate the distinct results with each other. To accomplish this a full simulation of molecular mobility on the basis of the single molecules would be required, taking reactions, interactions and geometrical restrictions into account. Such a simulation does not yet exist but is urgently needed in order to obtain a full view of the dynamic processes inside live cells.

Numerous interesting problems in biology and biophysics have been studied by single molecule techniques during the past few years [36, 37] but application to living cell systems is still in its infancy [38]. Single molecule microscopy and single particle tracking permit a completely new and fascinating view to intracellular dynamics. For the first time ever, this technique provides a direct, real-time insight into molecular processes in living cells with almost molecular resolution.

\section{Acknowledgements}

I gratefully acknowledge financial support by the Volkswagen Foundation, the German Research Foundation (DFG) and the Rheinische Friedrich-WilhelmsUniversität Bonn. I am indebted to Reiner Peters for a successful and long-standing cooperation; and I thank David Grünwald, Thorsten Kues and Jan Peter Siebrasse for a very successful cooperation during recent years. Finally, I thank Werner Wendler for preparing Figures 1.1 and 1.2, Andreas Hoekstra for preparing a first draft of Figure 1.3 and Jan Peter Siebrasse for a critical reading of the manuscript.

\section{References}

1 Donnert, G., Keller, J., Medda, R., Andrei, M.A., Rizzoli, S.O., Luhrmann, R., Jahn, R., Eggeling, C. and Hell, S.W. (2006) Macromolecular-scale resolution in biological fluorescence microscopy. Proceedings of the National Academy of Sciences of the United States of America, 103, 11440-11445. 
2 Heintzmann, R., Jovin, T.M. and Cremer, C. (2002) Saturated patterned excitation microscopy - a concept for optical resolution improvement. Journal of the Optical Society of America, 19, 1599-1609.

3 Gustafsson, M.G. (2005) Nonlinear structured-illumination microscopy: wide-field fluorescence imaging with theoretically unlimited resolution. Proceedings of the National Academy of Sciences of the United States of America, 102, 13081-13086.

4 Verkman, A.S. (2002) Solute and macromolecule diffusion in cellular aqueous compartments. Trends in Biochemical Sciences, 27, 27-33.

5 Enderlein, J., Gregor, I., Patra, D., Dertinger, T. and Kaupp, U.B. (2005) Performance of fluorescence correlation spectroscopy for measuring diffusion and concentration. Chemphyschem, $\mathbf{6}$, 2324-2336.

6 Haustein, E. and Schwille, P. (2007) Fluorescence correlation spectroscopy. novel variations of an established technique. Annual Review of Biophysics and Biomolecular Structure, 36, 151-169.

7 Kubitscheck, U. (2006) Fluorescence microscopy: single particle tracking, in Encyclopedic Reference of Genomics and Proteomics in Molecular Medicine (eds D. Ganten and K. Ruckpaul), Springer.

8 Yildiz, A., Forkey, J.N., McKinney, S.A., Ha, T., Goldman, Y.E. and Selvin, P.R. (2003) Myosin V walks hand-over-hand: single fluorophore imaging with $1.5-\mathrm{nm}$ localization. Science, 300, 2061-2065.

9 Kubitscheck, U., Kückmann, O., Kues, T. and Peters, R. (2000) Imaging and tracking of single GFP molecules in solution. Biophysical Journal, 78, 2170-2179.

10 Thompson, R.E., Larson, D.R. and Webb, W.W. (2002) Precise nanometer localization analysis for individual fluorescent probes. Biophysical Journal, 82, 2775-2783.
11 Grunwald, D., Hoekstra, A., Dange, T., Buschmann, V. and Kubitscheck, U. (2006) Direct observation of single protein molecules in aqueous solution. Chemphyschem, 7, 812-815.

12 Harms, G.S., Cognet, L., Lommerse, P.H., Blab, G.A., Kahr, H., Gamsjager, R., Spaink, H.P., Soldatov, N.M., Romanin, C. and Schmidt, T. (2001) Single-molecule imaging of l-type $\mathrm{Ca}(2+)$ channels in live cells. Biophysical Journal, 81, 2639-2646.

13 Kues, T., Peters, R. and Kubitscheck, U. (2001) Visualization and tracking of single protein molecules in the cell nucleus. Biophysical Journal, 80, 2954-2967.

14 Yang, W., Gelles, J. and Musser, S.M. (2004) Imaging of single-molecule translocation through nuclear pore complexes. Proceedings of the National Academy of Sciences of the United States of America, 101, 12887-12892.

15 Kubitscheck, U., Grunwald, D., Hoekstra, A., Rohleder, D., Kues, T., Siebrasse, J.P. and Peters, R. (2005) Nuclear transport of single molecules: dwell times at the nuclear pore complex. The Journal of Cell Biology, 168, 233-243.

16 Grunwald, D., Spottke, B., Buschmann, V. and Kubitscheck, U. (2006) Intranuclear binding kinetics and mobility of single U1 snRNP particles in living cells. Molecular Biology of the Cell, 17, 5017-5027.

17 Seisenberger, G., Ried, M.U., Endress, T., Buning, H., Hallek, M. and Brauchle, C. (2001) Real-time single-molecule imaging of the infection pathway of an adenoassociated virus. Science, 294, 1929-1932.

18 Babcock, H.P., Chen, C. and Zhuang, X. (2004) Using single-particle tracking to study nuclear trafficking of viral genes. Biophysical Journal, 87, 2749-2758.

19 Shav-Tal, Y., Darzacq, X., Shenoy, S.M., Fusco, D., Janicki, S.M., Spector, D.L. and Singer, R.H. (2004) Dynamics of single mRNPs in nuclei of living cells. Science, 304, 1797-1800.

20 Michalet, X., Pinaud, F.F., Bentolila, L.A., Tsay, J.M., Doose, S., Li, J.J., Sundaresan, G., Wu, A.M., Gambhir, S.S. and Weiss, S. 
(2005) Quantum dots for live cells, in vivo imaging, and diagnostics. Science, 307 , 538-544.

21 Bausinger, R., von Gersdorff, K., Braeckmans, K., Ogris, M., Wagner, E., Brauchle, C. and Zumbusch, A. (2006) The transport of nanosized gene carriers unraveled by live-cell imaging. Angewandte Chemie (International Edition in English), 45, 1568-1572.

22 Goulian, M. and Simon, S.M. (2000) Tracking single proteins within cells. Biophysical Journal, 79, 2188-2198.

23 Misteli, T. (2005) Concepts in nuclear architecture. Bioessays: News and Reviews in Molecular, Cellular and Developmental Biology, 27, 477-487.

24 Darzacq, X., Singer, R.H. and Shav-Tal, Y. (2005) Dynamics of transcription and mRNA export. Current Opinion in Cell Biology, 17, 332-339.

25 Will, C.L. and Luhrmann, R. (2001) Spliceosomal UsnRNP biogenesis, structure and function. Current Opinion in Cell Biology, 13, 290-301.

26 Medalia, O., Typke, D., Hegerl, R., Angenitzki, M., Sperling, J. and Sperling, R. (2002) Cryoelectron microscopy and cryoelectron tomography of the nuclear pre-mRNA processing machine. Journal of Structural Biology, 138, 74-84.

27 Lamond, A.I. and Spector, D.L. (2003) Nuclear speckles: a model for nuclear organelles. Nature Reviews. Molecular Cell Biology, 4, 605-612.

28 Kues, T., Dickmanns, A., Lührmann, R., Peters, R. and Kubitscheck, U. (2001) High intranuclear mobility and dynamic clustering of the splicing factor U1 snRNP observed by single particle tracking. Proceedings of the National Academy of Sciences of the United States of America, 98, 12021-12026.

29 Wachsmuth, M., Waldeck, W. and Langowski, J. (2000) Anomalous diffusion of fluorescent probes inside living cell nuclei investigated by spatially-resolved fluorescence correlation spectroscopy.
Journal of Molecular Biology, 298,

677-689.

30 Seksek, O., Biwersi, J. and Verkman, A.S. (1997) Translational diffusion of macromolecule-sized solutes in cytoplasm and nucleus. Journal of Cell Biology, 138, 131-142.

31 Gorisch, S.M., Richter, K., Scheuermann, M.O., Herrmann, H. and Lichter, P. (2003)

Diffusion-limited compartmentalization of mammalian cell nuclei assessed by microinjected macromolecules. Experimental Cell Research, 289, 282-294.

32 Phair, R.D. and Misteli, T. (2001) Kinetic modelling approaches to in vivo imaging. Nature Reviews. Molecular Cell Biology, 2, 898-907.

33 Voie, A.H., Burns, D.H. and Spelman, F.A. (1993) Orthogonal-plane fluorescence optical sectioning: three-dimensional imaging of macroscopic biological specimens. Journal of Microscopy, 170, 229-236.

34 Huisken, J., Swoger, J., Del Bene, F., Wittbrodt, J. and Stelzer, E.H. (2004) Optical sectioning deep inside live embryos by selective plane illumination microscopy. Science, 305, 1007-1009.

35 Engelbrecht, C.J. and Stelzer, E.H. (2006) Resolution enhancement in a lightsheet-based microscope (SPIM). Optics Letters, 31, 1477-1479.

36 Zlatanova, J. and van Holde, K. (2006) Single-molecule biology: what is it and how does it work? Molecular Cell, 24, 317-329.

37 Greenleaf, W.J., Woodside, M.T. and Block, S.M. (2007) High-resolution, singlemolecule measurements of biomolecular motion. Annual Review of Biophysics and Biomolecular Structure, 36, 171-190.

38 Peters, R. (2007) Single-molecule fluorescence analysis of cellular nanomachinery components. Annual Review of Biophysics and Biomolecular Structure, 36, 371-394.

39 Saxton, M. J. and Jocobsen, K. (1997) Single-particle tracking: applications to membrane dynamics. Annual Review of Biophysics and Biomolecular Structure, 26,373-399. 
